\title{
Steady-state entanglement in a double-well Bose-Einstein condensate through coupling to a superconducting resonator
}

\author{
H. T. $\mathrm{Ng}^{1}$ and Shih-I Chu ${ }^{1,2}$ \\ ${ }^{1}$ Center for Quantum Science and Engineering, Department of Physics, National Taiwan University, Taipei 10617, Taiwan \\ ${ }^{2}$ Department of Chemistry, University of Kansas, Lawrence, Kansas 66045, USA
}

(Received 30 May 2011; published 23 August 2011)

\begin{abstract}
We consider a two-component Bose-Einstein condensate in a double-well potential, where the atoms are magnetically coupled to a single mode of the microwave field inside a superconducting resonator. We find that the system has different dark-state subspaces in the strong- and weak-tunneling regimes. In the limit of weak tunnel coupling, steady-state entanglement between the two spatially separated condensates can be generated by evolving to a mixture of dark states via the dissipation of the photon field. We show that the entanglement can be faithfully indicated by an entanglement witness. Long-lived entangled states are useful for quantum-information processing with atom-chip devices.
\end{abstract}

DOI: 10.1103/PhysRevA.84.023629

PACS number(s): 03.75.Gg, 03.75.Lm, 42.50.Pq

\section{INTRODUCTION}

Recently, the realization of a Bose-Einstein condensate (BEC) strongly coupled to the quantized photon field in an optical cavity has been shown [1,2]. This paves the way to studying the interplay of atomic interactions and atom-photon interactions. For example, the novel quantum phase transition of a condensate coupled to a cavity has been demonstrated [3]. Strong atom-photon coupling is useful for quantum communications [4] such as the light-matter interface [1,2].

Alternatively, strong coupling of ultracold atoms to a superconducting resonator has been recently proposed [5]. The two long-lived hyperfine states $|e\rangle=\left|F=2, m_{F}=1\right\rangle$ and $|g\rangle=\left|F=1, m_{F}=-1\right\rangle$ of ${ }^{87} \mathrm{Rb}[6]$ are considered to be magnetically coupled to the microwave field via their magnetic dipoles [5,7]. Since the high- $Q$ superconducting resonator can be fabricated to a small mode volume [8] and the coupling strength can be greatly increased due to the collective enhancement [4], the strong coupling of ultracold atoms in the microwave regime can be achieved [5].

In this paper, we study a two-component BEC in a doublewell potential [9], where all atoms are equally coupled to a single mode of the microwave field inside a superconducting resonator. Two weakly linked condensates can be created in a magnetic double-well potential on an atom chip $[10,11]$ or in an optical double-well potential [12]. In fact, the tunneling dynamics between the atoms in two wells has been recently observed [13-15]. A double-well BEC coupled to an optical cavity has also been discussed in the literature [16-18]. However, the spontaneous emission rate of excited states used for optical transitions in experiments $[1,2]$ is much higher than the tunneling rate of the atoms between the two wells [13-15]. Here we consider the two hyperfine states $|e\rangle$ and $|g\rangle$ of ${ }^{87} \mathrm{Rb}$ with the transition frequency $2 \pi \times 6.8 \mathrm{GHz}$ [6]. The coherence times $[19,20]$ of these hyperfine spin states $(|e\rangle$ and $|g\rangle)$ are much longer than both the time scales of tunneling and atom-photon interactions. Therefore, this system offers possibilities for the study of how the tunnel couplings between the two spatially separated condensates affect the atom-photon dynamics.

We focus our investigation on the the system in the limits of the strong and weak tunnel couplings. We find that the system has different dark-state subspaces [21] in these two tunneling regimes. In the weak-tunneling regime, the system has a family of dark states which can be used for producing quantum entanglement between the condensates. Here we propose to efficiently generate steady-state entanglement between the two spatially separated condensates by evolving to a mixture of dark states through the dissipation of the photon field [22-24]. Note that our scheme does not require any adjustment of the tunneling strength. It is different from other methods [9] which depend on the strength of tunnel couplings to generate entanglement. In addition, the entanglement generated between the two condensates can be used for the implementation of quantum state transfer [25]. This may be useful for quantum-information processing with atom-chip devices [20].

This paper is organized as follows. In Sec. II, we introduce the system of a two-component condensate in a doublewell potential, and the two-level atoms are coupled to a superconducting resonator. In Sec. III, we derive the two effective Hamiltonians in the strong- and weak-tunneling regimes. In Sec. IV, we investigate the dark-state subspaces and the atom-photon dynamics in the two tunneling limits. In Sec. V, we provide a method to produce the steady-state entanglement between the two condensates in a double well. A summary is given in Sec. VI. In the Appendix, we discuss the validity of the effective Hamiltonian in the strong-tunneling regime.

\section{SYSTEM}

We consider a two-component BEC being trapped in a double-well potential [9], and the condensate is placed near the surface of a superconducting resonator as shown in Fig. 1. The atoms, with two internal states $|e\rangle$ and $|g\rangle$, are coupled to a single mode of the photon field via their magnetic dipoles.

\section{A. Two-component condensate trapped in a double-well potential}

We first introduce the system of a two-component condensate in a one-dimensional (1D) double-well potential which 


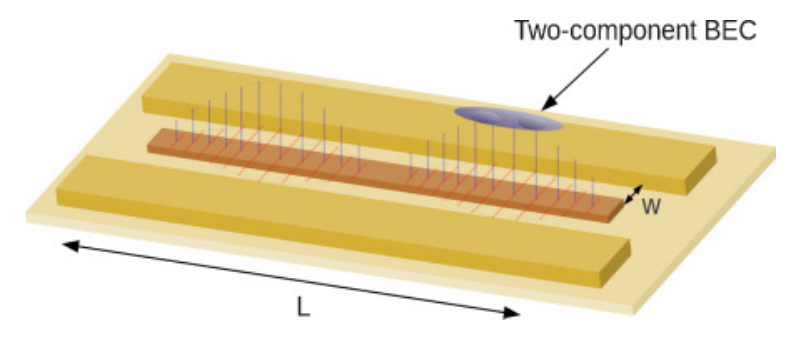

FIG. 1. (Color online) Schematic of a two-component BEC coupled to a single-mode of the photon field inside a superconducting resonator. A two-component condensate is trapped in a double-well potential, and it is placed close to the surface of the superconducting resonator. The atoms are coupled to the magnetic field via their magnetic dipoles. The parameters $L$ and $w$ are the length and width of the superconducting resonator, respectively.

can be described by the Hamiltonian as

$$
\begin{aligned}
H_{0}= & \sum_{\alpha} \int d x \Psi_{\alpha}^{\dagger}(x)\left[-\frac{\hbar^{2}}{2 m_{\alpha}} \frac{\partial^{2}}{\partial x^{2}}+V_{\mathrm{DW}}(x)\right. \\
& \left.+\tilde{U}_{\alpha} \Psi_{\alpha}^{\dagger}(x) \Psi_{\alpha}(x)\right] \Psi_{\alpha}(x) \\
& +2 \tilde{U}_{e g} \int d x \Psi_{e}^{\dagger}(x) \Psi_{g}^{\dagger}(x) \Psi_{g}(x) \Psi_{e}(x),
\end{aligned}
$$

where $\Psi_{\alpha}(x)$ is the field operator of the atoms for the internal state $|\alpha\rangle$ at the position $x$, and the indices $\alpha=g, e$ represent the ground and the excited states, respectively. Here $m_{\alpha}$ is the mass of the atom in the state $|\alpha\rangle$ and $V_{\mathrm{DW}}(x)$ is the 1D double-well potential which is given by [11]

$$
V_{\mathrm{DW}}(x)=V_{d}\left[1-\left(\frac{x}{x_{0}}\right)^{2}\right]^{2},
$$

where $V_{d}$ is the barrier height and $x_{0}$ is the distance between the two separate potential wells. The atoms are transversely confined in the $y$ and $z$ directions with the trap frequencies $\omega_{\perp}$. The size of the ground-state wave function in the transverse motion is $a_{\perp}=\sqrt{\hbar / m_{\alpha} \omega_{\perp}}$ [26,27], where $m_{e}$ and $m_{g}$ are nearly equal. Since the transverse frequencies are much larger than the trap frequency in the $x$ direction, the transverse motions of the atoms are frozen out. The parameters $\tilde{U}_{\alpha}$ and $\tilde{U}_{e g}$ are the effective 1D interaction strengths between the inter-, and the intracomponent condensates, as [26,27]

$$
\begin{gathered}
\tilde{U}_{\alpha}=\frac{2 \hbar^{2} a_{\alpha}}{m_{\alpha} a_{\perp}^{2}}\left(1-C \frac{a_{\alpha}}{\sqrt{2} a_{\perp}}\right)^{-1}, \\
\tilde{U}_{e g}=\frac{4 \hbar^{2} m_{e} m_{g} a_{e g}}{\left(m_{e}+m_{g}\right) a_{\perp}^{2}}\left(1-C \frac{a_{e g}}{\sqrt{2} a_{\perp}}\right)^{-1},
\end{gathered}
$$

where $C \approx 1.4603$. The parameters $a_{\alpha}$ and $a_{e g}$ are the threedimensional $s$-wave scattering lengths for the inter-, and the intracomponent condensates.

We adopt the two-mode approximation [28] such that the field operator $\Psi_{\alpha}(x)$ can be expanded in terms of the two localized mode functions $u_{\alpha_{L}}(x)$ and $u_{\alpha_{R}}(x)$ as

$$
\Psi_{\alpha}(x)=\alpha_{L} u_{\alpha L}(x)+\alpha_{R} u_{\alpha R}(x)
$$

where $\alpha_{L}$ and $\alpha_{R}$ are the annihilator operators of the atoms in the state $\alpha=e, g$ for the left and right modes of the double-well potential, respectively. The Hamiltonian of the system [9], within the two-mode approximation, can be written as

$$
\begin{aligned}
H_{0}^{\prime}= & \hbar E_{e}\left(e_{L}^{\dagger} e_{L}+e_{R}^{\dagger} e_{R}\right)+\hbar E_{g}\left(g_{R}^{\dagger} g_{R}+g_{L}^{\dagger} g_{L}\right) \\
& -\hbar J_{e}\left(e_{L}^{\dagger} e_{R}+e_{R}^{\dagger} e_{L}\right)-\hbar J_{g}\left(g_{L}^{\dagger} g_{R}+g_{R}^{\dagger} g_{L}\right) \\
& +\hbar U_{e e}\left[\left(e_{L}^{\dagger} e_{L}\right)^{2}+\left(e_{R}^{\dagger} e_{R}\right)^{2}\right]+\hbar U_{g g}\left[\left(g_{L}^{\dagger} g_{L}\right)^{2}\right. \\
& \left.+\left(g_{R}^{\dagger} g_{R}\right)^{2}\right]+2 \hbar U_{e g}\left(e_{L}^{\dagger} e_{L} g_{L}^{\dagger} g_{L}+e_{R}^{\dagger} e_{R} g_{R}^{\dagger} g_{R}\right)
\end{aligned}
$$

where

$$
\begin{gathered}
E_{\alpha}=\frac{1}{\hbar} \int d x u_{\alpha j}^{*}(x)\left[-\frac{\hbar^{2}}{2 m_{\alpha}} \frac{\partial^{2}}{\partial x^{2}}+V_{\mathrm{DW}}(x)\right] u_{\alpha j}(x), \\
J_{\alpha}=-\frac{1}{\hbar} \int d r u_{\alpha L}^{*}(x)\left[-\frac{\hbar^{2}}{2 m_{\alpha}} \frac{\partial}{\partial x^{2}}+V_{\mathrm{DW}}(x)\right] u_{\alpha R}(x), \\
U_{\alpha}=\frac{\tilde{U}_{\alpha}}{\hbar} \int d x\left|u_{\alpha j}(x)\right|^{4}, \\
U_{\alpha \beta}=\frac{\tilde{U}_{\alpha \beta}}{\hbar} \int d r\left|u_{\alpha j}(x)\right|^{2}\left|u_{\beta j}(x)\right|^{2},
\end{gathered}
$$

and $j=L, R$. The positive parameters $E_{\alpha}$ and $J_{\alpha}$ [29] are the ground-state frequencies of the localized mode $\alpha_{L, R}$, and the tunneling strengths between the two wells for the atoms in the states $\alpha$. Here $U_{\alpha}$ and $U_{\alpha \beta}$ are the two positive parameters which describe the inter- and intracomponent interaction strengths, respectively.

\section{B. Atoms coupled to the photon field in a microwave cavity}

We consider that the atoms are coupled to a single mode of the photon field via their magnetic dipoles [5]. Within the twomode approximation, the Hamiltonian, describing the system of cavity field, the atoms, and their interactions, is given by

$$
\begin{aligned}
H_{I}= & \hbar \omega_{a} a^{\dagger} a+\hbar \omega_{0}\left(e_{L}^{\dagger} e_{L}+e_{R}^{\dagger} e_{R}\right) \\
& +\hbar g\left[a\left(e_{L}^{\dagger} g_{L}+e_{R}^{\dagger} g_{R}\right)+\text { H.c. }\right],
\end{aligned}
$$

where $\omega_{a}$ and $a$ are the frequency and the annihilator operator of the single mode of the photon field, and $\omega_{0}$ is the transition frequency of the two internal states. Here we have assumed that the wavelength of the microwave field $(\sim 1 \mathrm{~cm})$ is much larger than the size of the condensate $(\sim 10 \mu \mathrm{m})[10,11]$. Therefore, all atoms are coupled to the photon field with the same coupling strength $g=\mu_{B} \sqrt{\mu_{0} \omega_{a} / 2 \hbar V}$ [7], where $\mu_{B}$ is the Bohr magneton, $\mu_{0}$ is the vacuum permeability, and $V$ is the volume of the superconducting resonator. The coupling strength $g$ can attain $1 \mathrm{kHz}$ [7] if the volume $V$ of the superconducting resonator is taken as $L \times w \times t_{h} \sim 1 \mathrm{~cm} \times 10 \mu \mathrm{m} \times 200 \mathrm{~nm}$ [7,8], where $L$ is the length, $w$ is the width, and $t_{h}$ is the thickness of the superconducting resonator.

\section{EFFECTIVE HAMILTONIANS IN STRONG- AND WEAK-TUNNELING REGIMES: LOW ATOMIC EXCITATIONS}

We will derive the effective Hamiltonians of the system in the limits of strong and weak tunnel couplings, where a few 
atomic excitations are only involved. Let us first write the total Hamiltonian of the system as

$$
\begin{aligned}
H= & \hbar \omega_{a} a^{\dagger} a+\hbar \omega_{0}\left(e_{L}^{\dagger} e_{L}+e_{R}^{\dagger} e_{R}\right)-\hbar J_{e}\left(e_{L}^{\dagger} e_{R}+e_{R}^{\dagger} e_{L}\right) \\
& -\hbar J_{g}\left(g_{L}^{\dagger} g_{R}+g_{R}^{\dagger} g_{L}\right)+\hbar U_{e e}\left[\left(e_{L}^{\dagger} e_{L}\right)^{2}+\left(e_{R}^{\dagger} e_{R}\right)^{2}\right] \\
& +\hbar U_{g g}\left[\left(g_{L}^{\dagger} g_{L}\right)^{2}+\left(g_{R}^{\dagger} g_{R}\right)^{2}\right]+2 \hbar U_{e g}\left(e_{L}^{\dagger} e_{L} g_{L}^{\dagger} g_{L}\right. \\
& \left.+e_{R}^{\dagger} e_{R} g_{R}^{\dagger} g_{R}\right)+\hbar g\left[a\left(e_{L}^{\dagger} g_{L}+e_{R}^{\dagger} g_{R}\right)+\text { H.c. }\right] .
\end{aligned}
$$

The total number of atoms $N$ is conserved. We have omitted the constant term $E_{0} N$ for a symmetric double well, where $E_{\alpha} \approx E_{0}$ for the two masses $m_{e}$ and $m_{g}$ being equal. It is convenient to work in the rotating frame by applying the unitary transformation to the Hamiltonian $H$ in Eq. (12), where the unitary operator $U(t)$ is

$$
U(t)=\exp \left[-i \omega_{a}\left(a^{\dagger} a+e_{L}^{\dagger} e_{L}+e_{R}^{\dagger} e_{R}\right) t\right]
$$

The transformed Hamiltonian becomes

$$
\begin{aligned}
H^{\prime}= & \hbar \Delta\left(e_{L}^{\dagger} e_{L}+e_{R}^{\dagger} e_{R}\right)-\hbar J_{e}\left(e_{L}^{\dagger} e_{R}+e_{R}^{\dagger} e_{L}\right) \\
& -\hbar J_{g}\left(g_{L}^{\dagger} g_{R}+g_{R}^{\dagger} g_{L}\right)+\hbar U_{e e}\left[\left(e_{L}^{\dagger} e_{L}\right)^{2}+\left(e_{R}^{\dagger} e_{R}\right)^{2}\right] \\
& +\hbar U_{g g}\left[\left(g_{L}^{\dagger} g_{L}\right)^{2}+\left(g_{R}^{\dagger} g_{R}\right)^{2}\right]+2 \hbar U_{e g}\left(e_{L}^{\dagger} e_{L} g_{L}^{\dagger} g_{L}\right. \\
& \left.+e_{R}^{\dagger} e_{R} g_{R}^{\dagger} g_{R}\right)+\hbar g\left[a\left(e_{L}^{\dagger} g_{L}+e_{R}^{\dagger} g_{R}\right)+\text { H.c. }\right], \quad
\end{aligned}
$$

where $\Delta=\omega_{0}-\omega_{a}$ is the detuning between the frequencies of the photon field and the two internal states.

In the strong-tunneling regime, the tunnel coupling is dominant and the strength of atom-atom interactions is relatively weak. On the contrary, in the weak-tunneling regime, the atom-atom interactions become dominant and the tunneling strength is negligible. We will show that these two cases exhibit the different behaviors in the atom-photon dynamics. We will provide derivations of the two effective Hamiltonians in the two tunneling limits in the following sections.

\section{A. Strong-tunneling regime}

In the limit of the strong tunnel coupling, the tunneling strengths are much larger than the strengths of the atom-atom interactions, i.e., $J_{e}, J_{g} \gg U_{e}, U_{g}, U_{e g}$. The total Hamiltonian of the system can be approximated as

$$
\begin{aligned}
H_{1}= & \hbar \Delta\left(e_{L}^{\dagger} e_{L}+e_{R}^{\dagger} e_{R}\right)-\hbar J_{e}\left(e_{L}^{\dagger} e_{R}+e_{R}^{\dagger} e_{L}\right) \\
& -\hbar J_{g}\left(g_{L}^{\dagger} g_{R}+g_{R}^{\dagger} g_{L}\right)+\hbar g\left[a\left(e_{L}^{\dagger} g_{L}+e_{R}^{\dagger} g_{R}\right)+\text { H.c. }\right]
\end{aligned}
$$

We have neglected the terms of the atom-atom interactions in this Hamiltonian.

The symmetric and asymmetric modes $g_{ \pm}$and $e_{ \pm}$can be related to the localized modes as

$$
\begin{aligned}
& g_{ \pm}=\frac{1}{\sqrt{2}}\left(g_{L} \pm g_{R}\right), \\
& e_{ \pm}=\frac{1}{\sqrt{2}}\left(e_{L} \pm e_{R}\right) .
\end{aligned}
$$

The Hamiltonian is then transformed as

$$
\begin{aligned}
H_{1}^{\prime}= & \hbar\left(\Delta-J_{e}\right) e_{+}^{\dagger} e_{+}+\hbar\left(\Delta+J_{e}\right) e_{-}^{\dagger} e_{-}-\hbar J_{g}\left(g_{+}^{\dagger} g_{+}-g_{-}^{\dagger} g_{-}\right) \\
& +\hbar g\left(a e_{+}^{\dagger} g_{+}+\text {H.c. }\right)+\hbar g\left(a e_{-}^{\dagger} g_{-}+\text {H.c. }\right) .
\end{aligned}
$$

Here the atoms are in symmetric (asymmetric) mode if they are populated in the states $g_{+}^{k}|0\rangle_{+}$or $e_{+}^{k}|0\rangle_{+}\left(g_{-}^{k}|0\rangle_{-}\right.$or $\left.e_{-}^{k}|0\rangle_{-}\right)$, where $|0\rangle_{+}\left(|0\rangle_{-}\right)$is the vacuum state of the symmetric (asymmetric) mode and $k$ is a non-negative integer.

We consider the system to be initially prepared in the ground state in the limit of strong tunnel coupling, i.e., the ground state of the symmetric mode. The ground state can be obtained by applying the operator $\left(g_{+}^{\dagger}\right)^{N}$ to the vacuum state $|0\rangle_{+}$of the symmetric mode, i.e.,

$$
\left|\Psi_{1}(0)\right\rangle=\frac{1}{\sqrt{N !}}\left(g_{+}^{\dagger}\right)^{N}|0\rangle_{+},
$$

where $N$ is the total number of atoms. Note that the atoms in the symmetric and asymmetric modes are independently coupled to the photon field in Eq. (18). Therefore, all atoms in the symmetric mode are only involved in the dynamics of the atom-photon interactions if the system starts with the state $\left|\Psi_{1}(0)\right\rangle$ in Eq. (19). In fact, there are only a few excitations in the asymmetric mode due to the atomic interactions. The effect of the excitations from the asymmetric mode to the dynamics of atom-photon interactions is very small. It is because the Rabi coupling strength cannot be greatly enhanced with a small number of atoms in the asymmetric mode. We briefly discuss the validity of this assumption in the Appendix.

It is instructive to express the Hamiltonian in terms of angular momentum operators:

$$
\begin{gathered}
S_{+}^{(+)}=g_{+} e_{+}^{\dagger}, \\
S_{-}^{(+)}=e_{+} g_{+}^{\dagger}, \\
S_{z}^{(+)}=\frac{1}{2}\left(e_{+}^{\dagger} e_{+}-g_{+}^{\dagger} g_{+}\right) .
\end{gathered}
$$

The Hamiltonian can be rewritten as

$$
\tilde{H}_{1}^{\prime}=\hbar \Delta S_{z}^{(+)}+\hbar g\left(a S_{+}^{(+)}+\text {H.c. }\right) \text {. }
$$

For simplicity, we have assumed that the tunneling strengths $J_{e}$ and $J_{g}$ are equal. We also have omitted the constant term $\hbar N \Delta / 2$.

By applying the Holstein-Primakoff transformation (HPT) [30], the angular momentum operators can be mapped onto the harmonic oscillators which are given by

$$
\begin{gathered}
S_{+}^{(+)}=b^{\dagger} \sqrt{N-b^{\dagger} b}, \\
S_{-}^{(+)}=b \sqrt{N-b^{\dagger} b}, \\
S_{z}^{(+)}=b^{\dagger} b-\frac{N}{2} .
\end{gathered}
$$

In the low degree of excitation, the mean excitation number $\left\langle b^{\dagger} b\right\rangle$ is much smaller than the total number of atoms $N$. The angular momentum operators can be approximated by the bosonic operators [9,31]. The effective Hamiltonian can be obtained as

$$
H_{\mathrm{eff}}^{(1)}=\hbar \Delta b^{\dagger} b+\hbar g \sqrt{N}\left(a b^{\dagger}+\text { H.c. }\right) .
$$

Note that the effective Rabi frequency is enhanced by a factor of $\sqrt{N}$. This effective Hamiltonian $H_{\text {eff }}^{(1)}$ in Eq. (27) describes the interaction between the collective-excitation mode and the single mode of the photon field. 


\section{B. Weak-tunneling regime}

Now we investigate the system in the weak-tunneling regime, where the atom-atom interaction strengths are much larger than the tunneling strengths, i.e, $U_{e}, U_{g}, U_{e g} \gg J_{e}, J_{g}$. In this limit, we assume that the tunneling between the two condensates is effectively turned off. The total Hamiltonian can be approximated as

$$
\begin{aligned}
H_{2}= & \hbar \Delta\left(e_{L}^{\dagger} e_{L}+e_{R}^{\dagger} e_{R}\right)+\hbar g\left[a\left(e_{L}^{\dagger} g_{L}+e_{R}^{\dagger} g_{R}\right)+\text { H.c. }\right] \\
& +\hbar U_{e e}\left[\left(e_{L}^{\dagger} e_{L}\right)^{2}+\left(e_{R}^{\dagger} e_{R}\right)^{2}\right]+\hbar U_{g g}\left[\left(g_{L}^{\dagger} g_{L}\right)^{2}+\left(g_{R}^{\dagger} g_{R}\right)^{2}\right] \\
& +2 \hbar U_{e g}\left(e_{L}^{\dagger} e_{L} g_{L}^{\dagger} g_{L}+e_{R}^{\dagger} e_{R} g_{R}^{\dagger} g_{R}\right) .
\end{aligned}
$$

Here we have ignored the terms of the tunnel couplings.

This Hamiltonian can be expressed in terms of the angular momentum operators:

$$
\begin{gathered}
S_{j+}=g_{j} e_{j}^{\dagger}, \\
S_{j-}=e_{j} g_{j}^{\dagger}, \\
S_{j z}=\frac{1}{2}\left(e_{j}^{\dagger} e_{j}-g_{j}^{\dagger} g_{j}\right),
\end{gathered}
$$

where $j=L, R$. Now the Hamiltonian is rewritten as

$$
\tilde{H}_{2}=\hbar \sum_{j=L, R}(\Delta+\delta) S_{j z}+\hbar g\left(a S_{j+}+\text { H.c. }\right)+\hbar \chi S_{j z}^{2} \text {, }
$$

where $\delta=\left(U_{e e}-U_{g g}\right) N / 2$ and $\chi=U_{e e}+U_{g g}-2 U_{e g}$. We have omitted the constant term $\hbar\left(U_{e e}+U_{g g}+2 U_{e g}\right) N^{2} / 16+$ $\hbar N \Delta / 2$ in Eq. (32).

We consider all atoms at the state $|g\rangle$ are initially prepared in the ground state of the Hamiltonian in Eq. (32), which can be described by a product of two number states as

$$
\left|\Psi_{2}(0)\right\rangle=|N / 2\rangle_{g_{L}}|N / 2\rangle_{g_{R}} .
$$

Without loss of generality, we assume that $N$ is an even number.

We apply the HPT such that the angular momentum operators can be mapped onto the harmonic oscillators as

$$
\begin{gathered}
S_{L+}=c^{\dagger} \sqrt{N / 2-c^{\dagger} c}, S_{L-}=c \sqrt{N / 2-c^{\dagger} c}, \\
S_{L z}=c^{\dagger} c-\frac{N}{4}, \\
S_{R+}=d^{\dagger} \sqrt{N / 2-d^{\dagger} d}, S_{R-}=d \sqrt{N / 2-d^{\dagger} d}, \\
S_{R z}=d^{\dagger} d-\frac{N}{4},
\end{gathered}
$$

If the mean numbers of the atomic excitations, $\left\langle c^{\dagger} c\right\rangle$ and $\left\langle d^{\dagger} d\right\rangle$, are much smaller than the number of atoms $N / 2$ in each well, then the Hamiltonian can be approximated [9,31] as

$$
\begin{aligned}
H_{\mathrm{eff}}^{(2)}= & \hbar \Delta_{w}\left(c^{\dagger} c+d^{\dagger} d\right)+\hbar g \sqrt{\frac{N}{2}}\left[a\left(c^{\dagger}+d^{\dagger}\right)+\text { H.c. }\right] \\
& +\hbar \chi\left[\left(c^{\dagger} c\right)^{2}+\left(d^{\dagger} d\right)^{2}\right],
\end{aligned}
$$

where $\Delta_{w}=2 \Delta+\delta-\chi N / 2$. The effective Rabi frequency is enhanced by a factor of $\sqrt{N / 2}$. The parameter $\chi$ is much smaller than the effective Rabi frequency because the scattering lengths of the inter- and intracomponent condensates of ${ }^{87} \mathrm{Rb}$ are very similar [6]. We will ignore the terms with the parameter $\chi$ in Eq. (38) in our later discussion.

The effective Hamiltonian $H_{\text {eff }}^{(2)}$ in Eq. (38) describes the interactions between the single mode of the photon field and the two modes of the collective excitations of the atoms in the left and right potential wells, respectively. This system can be described by a system of three coupled harmonic oscillators. The effective Rabi frequency for each atomic mode is proportional to the factor $\sqrt{N / 2}$. This is different from the effective Rabi frequency, in the strong-tunneling regime, which is proportional to the factor $\sqrt{N}$.

\section{DARK STATES AND QUANTUM DYNAMICS OF THE SYSTEM}

We now study dark states of the system which has different dark-state subspaces in the strong- and weak-tunneling regimes. Let us first introduce the definition of dark states. Dark states [21] are the eigenstates of the atom-photon interaction operator $\mathcal{V}$, with zero eigenvalues, i.e.,

$$
\mathcal{V} \mid \text { dark }\rangle=0 \mid \text { dark }\rangle=0 .
$$

Dark states, in the strong- and weak-tunneling regimes, in this system can be obtained as

$$
H_{\mathrm{eff}}^{(j)}|D\rangle_{j}=0
$$

where $H_{\mathrm{eff}}^{(j)}$ are the two effective Hamiltonians in Eqs. (27) and (38) with zero detunings $\left(\Delta=\Delta_{w}=0\right)$ and $j=1,2$.

In the limit of strong tunnel coupling, the dark state $|D\rangle_{1}$ is the product state of the vacuum state of the photon field and the ground state of the atomic mode $b$, which is given by

$$
|D\rangle_{1}=|0\rangle_{a}|0\rangle_{b}
$$

This state is the ground state of the coupled system of the atoms and the photon field.

In the weak-tunneling regime, the system has a family of dark states. The family of dark states are

$$
\left|D_{n}\right\rangle_{2}=|0\rangle_{a}\left|D_{n}^{a}\right\rangle
$$

where

$$
\left|D_{n}^{a}\right\rangle=2^{-n / 2} \sum_{j=0}^{n}(-1)^{j} \sqrt{C_{j}^{n}}|n-j\rangle_{c}|j\rangle_{d},
$$

and $C_{j}^{n}$ is the binomial coefficient. The dark states $\left|D_{n}\right\rangle_{2}$ are the product state of the vacuum state $|0\rangle_{a}$ of the photon field and the states $\left|D_{n}^{a}\right\rangle$ are the eigenstates of the operator $c+d$ with zero eigenvalues. Note that the states $\left|D_{n}^{a}\right\rangle$ in Eq. (43) are a superposition of the states $|n-j\rangle_{c}|j\rangle_{d}$ which have the same degree of atomic excitations.

To gain more insight into dark states, let us first investigate the atom-photon dynamics subject to the dissipation of the photon field. A superconducting resonator with the frequency $\sim 40 \mathrm{GHz}$ can be cooled down to low temperatures $(\sim 25 \mathrm{mK})$ [32]. This allows us to consider the cavity field being weakly coupled to the reservoir at zero temperature [33]. Note that the relaxation time (several $\mu \mathrm{s}$ ) of the single photon inside the superconducting resonator is much shorter than the coherence 

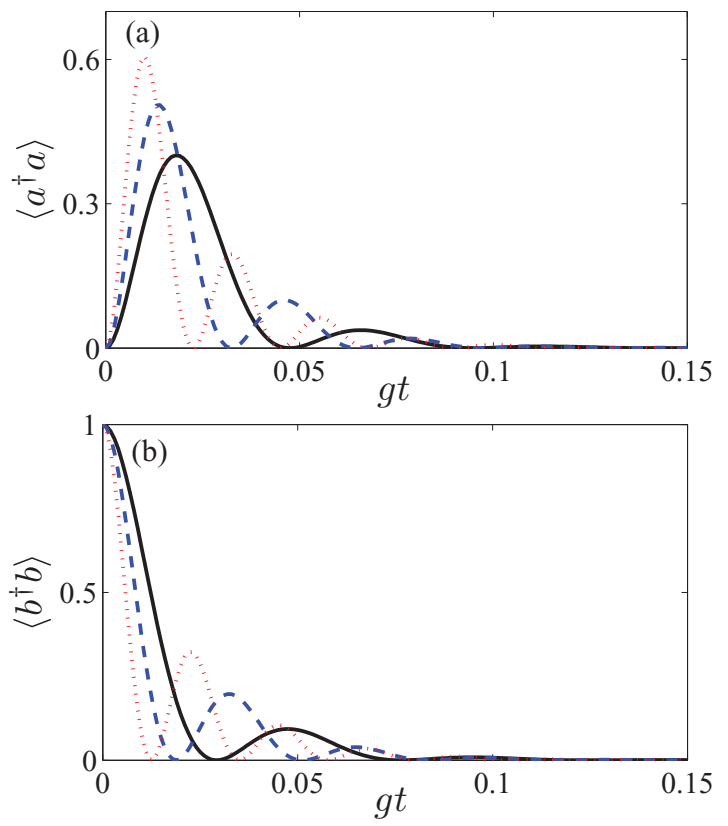

FIG. 2. (Color online) Time evolution of the mean photon number (a) and the mean atomic excitations (b) with the damping rate $\kappa=100 \mathrm{~g}$ and the detuning $\Delta=0$. The different number of atoms $N$ are $5 \times 10^{3}$ (black solid line), $1 \times 10^{4}$ (blue dashed line), and $2 \times 10^{4}$ (red dotted line).

time $(\sim 1 s)$ of the cold atoms $[19,20]$. The effect of the dissipation of the atoms caused by the noise of the surface of the superconductor is negligible [34]. The main source of the dissipation is the damping of the photon field. The dynamics of the system can be described by the master equation, for zero temperature, as $[33,35]$

$$
\dot{\rho}_{j}=-\frac{i}{\hbar}\left[H_{\mathrm{eff}}^{(j)}, \rho_{j}\right]+\frac{\kappa}{2}\left(2 a \rho_{j} a^{\dagger}-a^{\dagger} a \rho_{j}-\rho_{j} a^{\dagger} a\right),
$$

where $\rho_{j}$ is the density matrix of the total system, and $j=$ 1,2 . Obviously, the dark states $|D\rangle_{1}$ and $\left|D_{n}\right\rangle_{2}$ are the steadystate solutions of the master equation in Eq. (44). Thus, the dark states are robust against the dissipation of the photon field. In the strong-tunneling regime, the steady state is the dark state $|D\rangle_{1}$. In the weak-tunneling regime, the state of the condensates evolves as a mixture of dark states $\left|D_{n}\right\rangle_{2}$ through the dissipation of the photon field.

Now we study the dynamics of the system in the strongtunneling regime, where the state is prepared as $|0\rangle_{a}|1\rangle_{b}$, and $|1\rangle_{b}$ is a number state. We plot the time of evolution of the mean photon number and mean atomic-excitation number in Fig. 2. The mean photon number and mean atomic excitations undergo a few oscillations and then both of them decay to zero. We also see that the faster rate of oscillations can be obtained if a larger number of atoms $N$ are used.

We proceed to investigate the atom-photon dynamics in the weak-tunneling regime. The system is initially prepared as the state $|0\rangle_{a}|1\rangle_{c}|0\rangle_{d}$, where $|1\rangle_{c}$ is a number state. In Fig. 3, we plot the mean photon number, and the mean excitation numbers of the two atomic modes versus time. When the atom-photon interactions are turned on, the excitation number of the atomic mode $c$ decreases while the mean photon number increases as shown in Fig. 3. Afterward, the mean excitation number
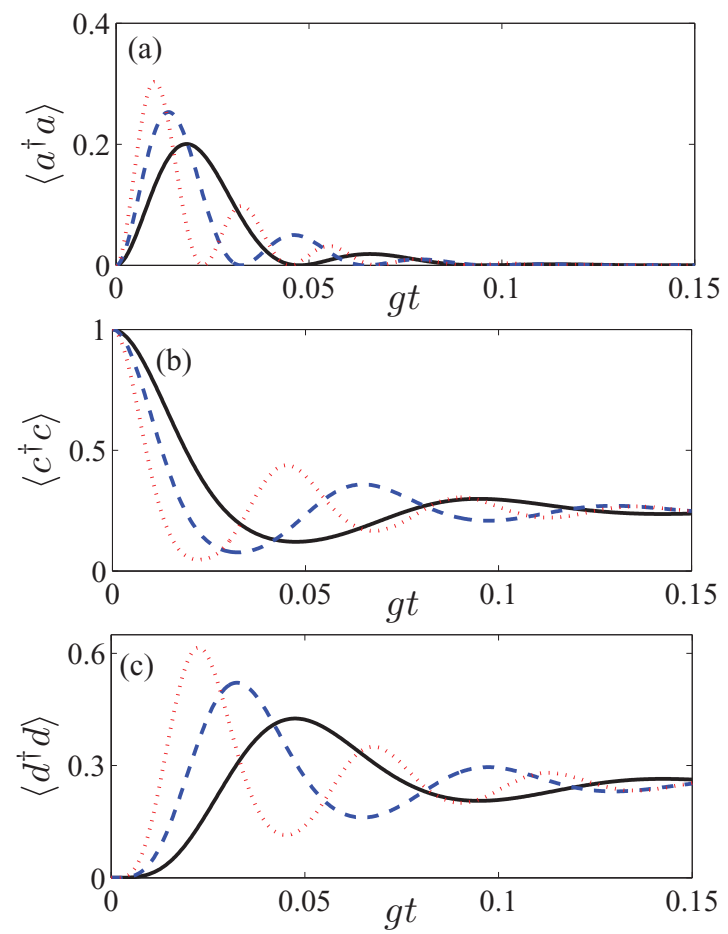

FIG. 3. (Color online) Dynamics of the mean photon number and mean atomic excitation numbers with the damping rate $\kappa=100 \mathrm{~g}$ and the detuning $\Delta_{w}=0$. (a) Mean photon number $\left\langle a^{\dagger} a\right\rangle$ as a function of the time $g t$. Time evolution of the mean atomic excitation numbers of the atomic mode $c$, in (b), and the atomic mode $d$, in (c). The different number of atoms $N$ are $5 \times 10^{3}$ (black solid line), $1 \times 10^{4}$ (blue dashed line) and $2 \times 10^{4}$ (red dotted line).

of the atomic mode $d$ starts to increase. This means that the energy of the atomic mode $c$ transfers to the photon field and the atomic mode $d$ absorbs the energy from the photon field. In this way, the two atomic modes exchange the energy via the photon field. The faster rate of exchanging energy between the atoms and the photon field can be attained if a larger number of atoms $N$ are used. We also note that the mean photon number in Fig. 3(a) is about half of the mean photon number in Fig. 2(a). It is because the atoms in the atomic mode $d$, in the weak-tunneling regime, absorbs the energy from the photon field.

In Fig. 3(a), the mean photon number decays to zero after a period of time. However, the mean excitation numbers of modes $c$ and $d$ remain nonzero as shown in Figs. 3(b) and 3 (c), because the state of the atoms evolves to a mixture of dark states $\left|D_{0}\right\rangle_{2}$ and $\left|D_{1}\right\rangle_{2}$, and a single excitation is shared by the atoms in the dark state $\left|D_{1}\right\rangle_{2}$. This results in the nonzero excitation numbers of the two atomic modes.

\section{GENERATION OF ENTANGLEMENT BETWEEN TWO SPATIALLY SEPARATED CONDENSATES}

We have shown that the system has different dark-state subspaces in the two tunneling limits. Now we study the entanglement between the condensates in the two different potential wells in the weak-tunneling regime. In this regime, the system has a family of dark states which can be used for generating entanglement. Here we consider the tunneling 

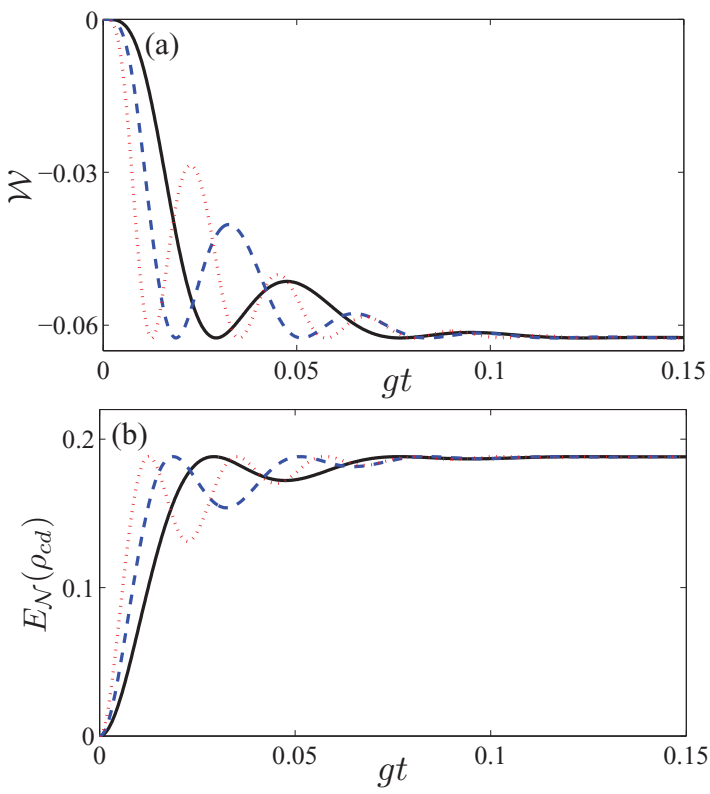

FIG. 4. (Color online) Time evolution of the entanglement witness in (a) and logarithmic negativity in (b), for the damping rate $\kappa=100 \mathrm{~g}$ and the detuning $\Delta_{w}=0$. The different number of atoms $N$ are $5 \times 10^{3}$ (black solid line), $1 \times 10^{4}$ (blue dashed line), and $2 \times 10^{4}$ (red dotted line).

between the wells to be effectively turned off. Therefore, the two independent condensates in the two potential wells are initially unentangled. We will show that steady-state entanglement between the two condensates can be produced by evolving to a mixture of dark states $\left\{\left|D_{n}\right\rangle_{2}\right\}$ through the dissipation of the photon field [22-24].

To study the quantum entanglement between the two atomic modes $c$ and $d$, it is necessary to obtain the density matrix of the atomic condensate. By tracing out the system of the photon field, we can obtain the density matrix $\rho_{c d}$,

$$
\rho_{c d}=\operatorname{Tr}_{a}(\rho),
$$

where $\rho$ is the density matrix of the total system. Let us first examine the entanglement of a single dark state $\left|D_{n}\right\rangle_{2}$. For a dark state $\left|D_{n}\right\rangle_{2}$ in Eq. (42), the density matrix $\rho_{c d}$ is given by

$$
\rho_{c d}=\left|D_{n}^{a}\right\rangle\left\langle D_{n}^{a}\right|
$$

where $\left|D_{n}^{a}\right\rangle$ is the state in Eq. (43). The degree of entanglement between the two atomic modes can be quantified by the von Neumann entropy. It is defined as

$$
E_{F}=-\operatorname{Tr}\left(\rho_{c} \ln \rho_{c}\right),
$$

where $\rho_{c}=\operatorname{Tr}_{d}\left(\rho_{c d}\right)$ is the reduced density matrix. The von Neumann entropy is

$$
E_{F}=-2^{-n} \sum_{j=0}^{n} C_{j}^{n} \ln \left|2^{-n} C_{j}^{n}\right| .
$$

Thus, the state $\left|D_{n}^{a}\right\rangle$ is an entangled state. The degree of twomode entanglement becomes higher for larger $n$.

In general, this density matrix $\rho_{c d}$ is a mixed state. To quantify the entanglement of a mixed state, logarithmic
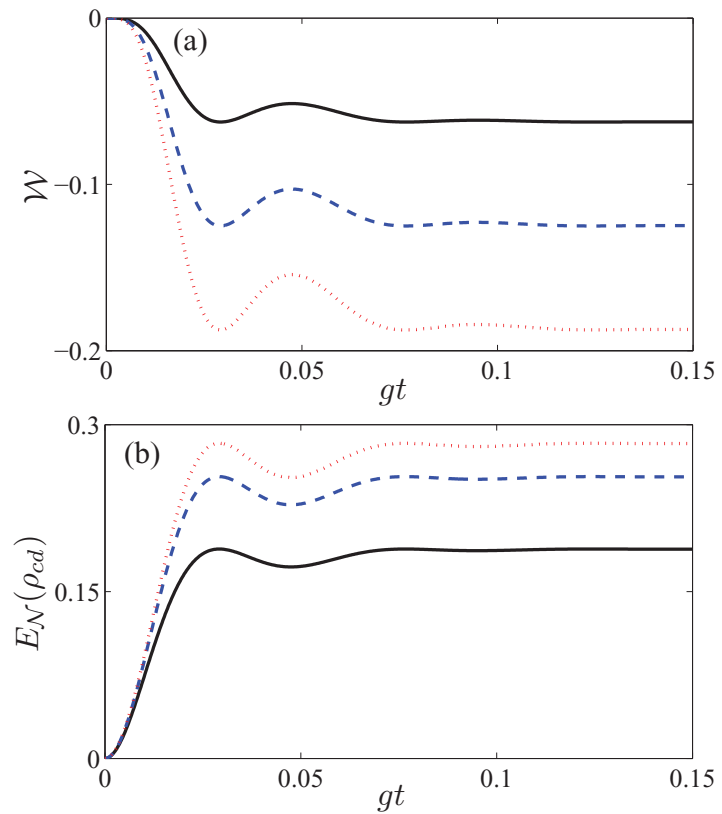

FIG. 5. (Color online) Dynamics of entanglement. (a) Entanglement witness $\mathcal{W}$ and (b) logarithmic negativity $E_{\mathcal{N}}\left(\rho_{c d}\right)$ as a function of time $g t$. The initial state $|0\rangle_{a}|n\rangle_{c}|0\rangle_{d}$ with the different excitation numbers $n$ are shown for $n=1$ (black solid line), $n=2$ (blue dashed line), and $n=3$ (red dotted line). The parameters are $\kappa=100 g$, $\Delta_{w}=0$, and $N=5 \times 10^{3}$.

negativity can be used [36]. The definition of logarithmic negativity is [36]

$$
E_{\mathcal{N}}\left(\rho_{c d}\right)=\log _{2}\left\|\rho_{c d}^{T_{c}}\right\|,
$$

where $\rho_{c d}^{T_{c}}$ is the partial transpose of the density matrix $\rho_{c d}$ and $\|\cdot\|$ is the trace norm.

However, logarithmic negativity is difficult to be experimentally determined. It is very useful to study an experimentally accessible quantity to detect the quantum entanglement between the two bosonic modes [37]. If an inequality

$$
\left|\left\langle c d^{\dagger}\right\rangle\right|^{2}>\left\langle n_{c} n_{d}\right\rangle
$$

is satisfied [37], then the state is an entangled state. Here $n_{c}=c^{\dagger} c$ and $n_{d}=d^{\dagger} d$ are the number operators of the atomic modes $c$ and $d$, respectively. For convenience, this quantity $\mathcal{W}$ is defined as

$$
\mathcal{W}=\left\langle n_{c} n_{d}\right\rangle-\left|\left\langle c d^{\dagger}\right\rangle\right|^{2} .
$$

If $\mathcal{W}$ is negative, then the state is nonseparable. This quantity $\mathcal{W}$ is called an entanglement witness [38].

We investigate the dynamics of entanglement between the two atomic modes. We consider an initial state as a product state of the three modes, i.e., $|0\rangle_{a}|1\rangle_{c}|0\rangle_{d}$, where $|1\rangle_{c}$ is a number state. We plot the entanglement witness and logarithmic negativity versus time as shown in Fig. 4. This figure shows that the entanglement witness decreases and logarithmic negativity increases with a similar rate, and then they saturate after a short time. This shows that the steady-state entanglement can be produced in a short time via the dissipative photon field. The entanglement can also be produced faster if a larger number of atoms are used. Besides, 
we can see that the entanglement witness is consistent with the logarithmic negativity to indicate the degree of entanglement. The entanglement witness is a faithful indicator for detecting entanglement between the two bosonic modes.

Next, we study the generation of entanglement by using an initial state $|0\rangle_{a}|n\rangle_{c}|0\rangle_{d}$ with a higher degree of excitation, where $|n\rangle_{c}$ is a number state and $n$ is larger than unity. In Fig. 5, the entanglement witness and logarithmic negativity are plotted versus time. It shows that a higher degree of entanglement can be obtained if higher excitation numbers $n=2,3$ are used.

\section{SUMMARY}

We have studied a two-component condensate in a doublewell potential, where the atoms are magnetically coupled to a single mode of the photon field inside a superconducting resonator. The system has different dark-state subspaces in the strong- and weak-tunneling regimes, , and it gives rise to different dynamics of atomic excitations in the two regimes. Steady-state entanglement between the two spatially separated condensates can be produced by evolving to a mixture of dark states through the dissipative photon field. We have shown that entanglement can be faithfully indicated by an entanglement witness.

\section{ACKNOWLEDGMENTS}

H.T.N. thanks David Hallwood for his careful reading and helpful comments, and C. K. Law for his useful discussion. This work was partially supported by the U.S. National Science Foundation. We also acknowledge the partial support of the National Science Council of Taiwan (Grant No. 100-2119M-002-013- MY3) and National Taiwan University (Grant No. 10R80700).

\section{APPENDIX : VALIDITY OF THE EFFECTIVE HAMILTONIAN IN THE STRONG-TUNNELING REGIME}

In this appendix, we examine the validity of the effective Hamiltonian $H_{\mathrm{eff}}^{(1)}$ in Eq. (27) in the limit of strong tunnel coupling. We express the Hamiltonian in term of the symmetric-mode and asymmetric-mode operators as

$$
\begin{aligned}
\tilde{H}= & \hbar\left(\Delta-J_{e}\right) e_{+}^{\dagger} e_{+}+\hbar\left(\Delta+J_{e}\right) e_{-}^{\dagger} e_{-}-\hbar J_{g}\left(g_{+}^{\dagger} g_{+}-g_{-}^{\dagger} g_{-}\right)+\hbar g\left(a e_{+}^{\dagger} g_{+}+\text {H.c. }\right)+\hbar g\left(a e_{-}^{\dagger} g_{-}\right. \\
& + \text {H.c. })+\frac{\hbar U_{e e}}{2}\left[\left(e_{+}^{\dagger} e_{+}+e_{-}^{\dagger} e_{-}\right)^{2}+\left(e_{+}^{\dagger} e_{-}+e_{-}^{\dagger} e_{+}\right)^{2}\right]+\frac{\hbar U_{g g}}{2}\left[\left(g_{+}^{\dagger} g_{+}+g_{-}^{\dagger} g_{-}\right)^{2}+\left(g_{+}^{\dagger} g_{-}\right.\right. \\
& \left.\left.+g_{-}^{\dagger} g_{+}\right)^{2}\right]+\hbar U_{e g}\left[\left(e_{+}^{\dagger} e_{+}+e_{-}^{\dagger} e_{-}\right)\left(g_{+}^{\dagger} g_{+}+g_{-}^{\dagger} g_{-}\right)+\left(e_{+}^{\dagger} e_{-}+e_{-}^{\dagger} e_{+}\right)\left(g_{+}^{\dagger} g_{-}+g_{-}^{\dagger} g_{+}\right)\right] .
\end{aligned}
$$

Let us define

$$
\begin{aligned}
F^{+} & =g_{-}^{\dagger} g_{+}, F^{-}=g_{+}^{\dagger} g_{-}, \\
F_{3} & =\frac{1}{2}\left(g_{-}^{\dagger} g_{-}-g_{+}^{\dagger} g_{+}\right) .
\end{aligned}
$$

The commutation relations $\left[F_{3}, F_{ \pm}\right]= \pm F_{ \pm}$and $\left[F_{+}, F_{-}\right]=$ $2 F_{z}$ are satisfied. The operators $F_{ \pm}$and $F_{z}$, and $S_{ \pm}^{(+)}$and $S_{z}^{(+)}$ in Eqs. (20) to (22) generate an su(3) algebra [39]. In the limit of large $N$, we can apply the HPT to the operators:

$$
\begin{gathered}
F^{+}=f^{\dagger} \sqrt{N-f^{\dagger} f}, F^{-}=f \sqrt{N-f^{\dagger} f}, \\
F_{3}=f^{\dagger} f-N / 2 .
\end{gathered}
$$

Assuming that the mean excitation number $\left\langle f^{\dagger} f\right\rangle$ is much smaller than $N$, we can approximate the operators as [39]

$$
f^{\dagger}=\frac{1}{\sqrt{N}} F^{+}, f=\frac{1}{\sqrt{N}} F^{-} .
$$

In the low-degree-of-excitation regime, the approximated Hamiltonian can be written as

$$
\tilde{H} \approx \hbar \omega_{a} a^{\dagger} a+\hbar \omega^{\prime} b^{\dagger} b+\hbar g \sqrt{N}\left(a b^{\dagger}+\text { H.c. }\right)+\tilde{H}^{\prime} .
$$

The Hamiltonian $\tilde{H}^{\prime}$ contains the terms of the operators in the asymmetric mode and the terms from the nonlinear interactions, and the constant terms are omitted, which can be written as

$$
\begin{aligned}
\tilde{H}^{\prime}= & \hbar J_{g} f^{\dagger} f+\frac{\hbar U_{g g} N}{2}\left(f^{\dagger}+f\right)^{2}+\hbar\left(\Delta+J_{e}\right)\left(e_{-}^{\dagger} e_{-}-e_{-}^{\dagger} e_{-}\right)+\hbar g\left(a e_{-}^{\dagger} g_{-}+\text {H.c. }\right) \\
& +\frac{\hbar U_{e e}}{2}\left[\left(e_{+}^{\dagger} e_{+}+e_{-}^{\dagger} e_{-}\right)^{2}+\left(e_{+}^{\dagger} e_{-}+e_{-}^{\dagger} e_{+}\right)^{2}\right]+\frac{\hbar U_{g g}}{2}\left(g_{+}^{\dagger} g_{+}+g_{-}^{\dagger} g_{-}\right)^{2} \\
& +\hbar U_{e g}\left[\left(e_{+}^{\dagger} e_{+}+e_{-}^{\dagger} e_{-}\right)\left(g_{+}^{\dagger} g_{+}+g_{-}^{\dagger} g_{-}\right)+\sqrt{N}\left(e_{+}^{\dagger} e_{-}+e_{-}^{\dagger} e_{+}\right)\left(f^{\dagger}+f\right)\right] .
\end{aligned}
$$




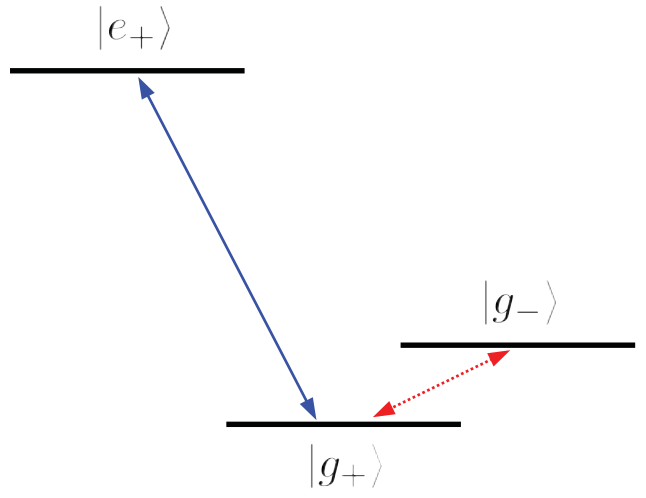

FIG. 6. (Color online) Level scheme of the atoms in the doublewell potential. In the strong-tunneling regime, the atoms, with the two states $\left|e_{+}\right\rangle$and $\left|g_{+}\right\rangle$, in the symmetric mode are coupled to the cavity field. The two ground states of the symmetric and asymmetric modes $\left(\left|g_{+}\right\rangle\right.$and $\left.\left|g_{-}\right\rangle\right)$are coupled to each other via the atom-atom interactions.

Here we consider the number of atoms in the excited states to be very small. We also assume that the strength of the Rabi coupling $g$ is weak compared to the tunneling strength $J_{g}$ and nonlinear strength $U_{g g} N$, but $g$ is much stronger than $U_{e e}, U_{g g}$, and $U_{e g}$. Therefore, the Hamiltonian $\tilde{H}^{\prime}$ can be approximated by the Hamiltonian $H^{\prime \prime}$ as

$$
\tilde{H}^{\prime \prime}=\hbar \lambda_{1} f^{\dagger} f+\hbar \lambda_{2}\left(f^{\dagger 2}+f^{2}\right),
$$

where

$$
\begin{array}{r}
\lambda_{1}=J_{g}+U_{g g} N, \\
\lambda_{2}=\frac{U_{g g} N}{2} .
\end{array}
$$

From Eq. (A7), nonlinear interactions can give rise to the transitions of the atoms in the symmetric mode to the atoms in the asymmetric mode, and vice versa. The level scheme is shown in Fig. 6. Note that this Hamiltonian $\tilde{H}^{\prime \prime}$ is exactly solvable. The time-evolution operator can be factorized as [35]

$$
\begin{aligned}
S(t)= & \exp \left(-i \tilde{H}^{\prime \prime} t / \hbar\right), \\
= & \exp \left(\frac{\Lambda_{2}}{2} f^{\dagger 2}\right) \exp \left[\frac{\ln \left(\Lambda_{1}\right)}{4}\left(f^{\dagger} f+f f^{\dagger}\right)\right] \\
& \times \exp \left(\frac{\Lambda_{2}}{2} f^{2}\right),
\end{aligned}
$$

where

$$
\Lambda_{1}=\left(\cosh \beta-\frac{\lambda_{1}^{\prime}}{2 \beta} \sinh \beta\right)^{-2}
$$

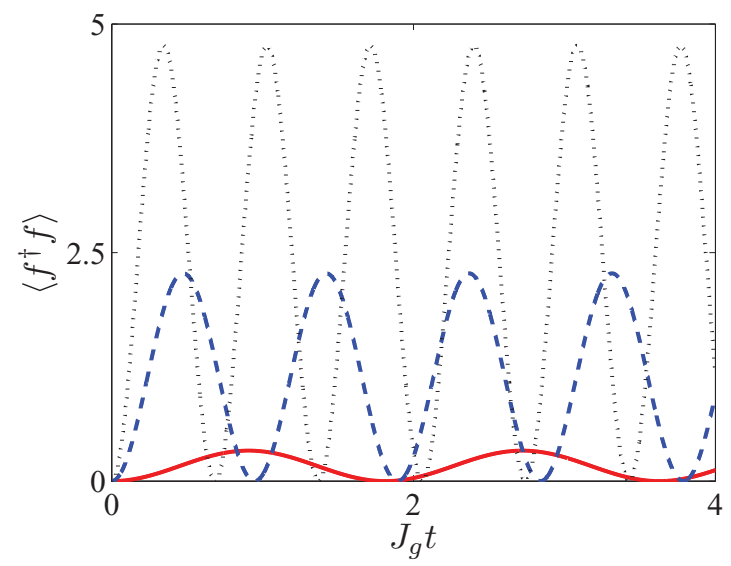

FIG. 7. (Color online) Expectation value $\left\langle f^{\dagger} f\right\rangle$ as a function of the time $J_{g} t$. Different strengths of $U_{g g} N$ are shown: $U_{g g} N=J_{g}$ (red solid line), $U_{g g} N=5 J_{g}$ (blue dashed line), and $U_{g g} N=10 J_{g}$ (black dotted line).

$$
\begin{gathered}
\Lambda_{2}=\frac{2 \lambda_{2}^{\prime} \sinh \beta}{2 \beta \cosh \beta-\lambda_{1}^{\prime} \sinh \beta}, \\
\beta^{2}=\frac{\lambda_{1}^{\prime 2}}{4}-\lambda_{2}^{\prime 2}, \\
\lambda_{1}^{\prime}=-2 i \lambda_{1} t, \lambda_{2}^{\prime}=-2 i \lambda_{2} t .
\end{gathered}
$$

We then apply the time-evolution operator $S(t)$ to the vacuum state $|0\rangle_{f}$ of the mode $f$. The state becomes

$$
\left|\Psi_{s}(t)\right\rangle=\Lambda_{1}^{1 / 4} \sum_{n=0}^{\infty} \frac{\sqrt{(2 n) !}}{n !}\left(\frac{\Lambda_{2}}{2}\right)^{n}|2 n\rangle_{f} .
$$

The mean excitation number $\left\langle f^{\dagger} f\right\rangle$ is

$$
\left\langle f^{\dagger} f\right\rangle=\left|\Lambda_{1}^{1 / 4}\right|^{2} \sum_{n=0}^{\infty} \frac{n(2 n) ! \Lambda_{2}^{2 n}}{2^{2 n-1}(n !)^{2}} .
$$

In Fig. 7, we plot the expectation value $\left\langle f^{\dagger} f\right\rangle$ versus the time, for the different strengths of atomic interaction $U_{g g} N$. It is shown that there are only a few excitations in the asymmetric mode even if the atomic-interaction strength $U_{g g} N$ is much larger than the tunneling strength $J_{g}$. Therefore, the Rabi coupling strength cannot be greatly increased due to the collective enhancement.
[1] Y. Colombe, T. Steinmetz, G. Dubois, F. Linke, D. Hunger, and J. Reichel, Nature (London) 450, 272 (2007).

[2] F. Brennecke, T. Donner, S. Ritter, T. Bourdel, M. Käohl, and T. Esslinger, Nature (London) 450, 268 (2007).

[3] K. Baumann, C. Guerlin, F. Brennecke, and T. Esslinger, Nature (London) 464, 1301 (2010).

[4] L.-M. Duan, M. Lukin, I. Cirac, and P. Zoller, Nature (London) 414, 413 (2001).
[5] K. Henschel, J. Majer, J. Schmiedmayer, and H. Ritsch, Phys. Rev. A 82, 033810 (2010).

[6] M. R. Matthews, D. S. Hall, D. S. Jin, J. R. Ensher, C. E. Wieman, E. A. Cornell, F. Dalfovo, C. Minniti, and S. Stringari, Phys. Rev. Lett. 81, 243 (1998).

[7] A. Imamoglu, Phys. Rev. Lett. 102, 083602 (2009).

[8] H. Wang et al., Appl. Phys. Lett. 95, 233508 (2009). 
[9] H. T. Ng, C. K. Law, and P. T. Leung, Phys. Rev. A 68, 013604 (2003); H. T. Ng and P. T. Leung, ibid. 71, 013601 (2005).

[10] T. Schumm et al., Nature Phys. 1, 57 (2005).

[11] K. Maussang, G. E. Marti, T. Schneider, P. Treutlein, Y. Li, A. Sinatra, R. Long, J. Esteve, and J. Reichel, Phys. Rev. Lett. 105, 080403 (2010).

[12] Y. Shin, G.-B. Jo, M. Saba, T. A. Pasquini, W. Ketterle, and D. E. Pritchard, Phys. Rev. Lett. 95, 170402 (2005).

[13] M. Albiez, R. Gati, J. Fölling, S. Hunsmann, M. Cristiani, and M. K. Oberthaler, Phys. Rev. Lett. 95, 010402 (2005)

[14] S. Fölling et al., Nature (London) 448, 1029 (2007).

[15] S. Trotzky et al., Science 319, 295 (2008).

[16] J. M. Zhang, W. M. Liu, and D. L. Zhou, Phys. Rev. A 77, 033620 (2008); 78, 043618 (2008).

[17] W. Chen and P. Meystre, Phys. Rev. A 79, 043801 (2009).

[18] J. Larson and J.-P. Martikainen, Phys. Rev. A 82, 033606 (2010).

[19] D. M. Harber, H. J. Lewandowski, J. M. McGuirk, and E. A. Cornell, Phys. Rev. A 66, 053616 (2002).

[20] P. Treutlein et al., Fortschr. Phys. 54, 702 (2006).

[21] M. Fleischhauer and M. D. Lukin, Phys. Rev. A 65, 022314 (2002).

[22] G. J. Yang, O. Zobay, and P. Meystre, Phys. Rev. A 59, 4012 (1999).

[23] M. B. Plenio, S. F. Huelga, A. Beige, and P. L. Knight, Phys. Rev. A 59, 2468 (1999).

[24] C. Joshi, A. Hutter, F. E. Zimmer, M. Jonson, E. Andersson, and P. Öhberg, Phys. Rev. A 82, 043846 (2010).
[25] S. Bose, Phys. Rev. Lett. 91, 207901 (2003).

[26] M. Olshanii, Phys. Rev. Lett. 81, 938 (1998).

[27] A. C. Pflanzer, S. Zöllner, and P. Schmelcher, Phys. Rev. A 81, 023612 (2010).

[28] G. J. Milburn, J. Corney, E. M. Wright, and D. F. Walls, Phys. Rev. A 55, 4318 (1997).

[29] Here the tunneling strengths $J_{\alpha}$ are assumed to be positive. In fact, the tunneling strengths can be negative for strong nonlinear-interaction strengths and the large barrier height. See the reference: D. Ananikian and T. Bergeman, Phys. Rev. A 73, 013604 (2006).

[30] T. Holstein and H. Primakoff, Phys. Rev. 58, 1098 (1949).

[31] H. T. Ng and K. Burnett, Phys. Rev. A 75, 023601 (2007).

[32] M. Hofheinz et al., Nature (London) 459, 546 (2009).

[33] H. T. Ng and F. Nori, Phys. Rev. A 82, 042317 (2010).

[34] B. Kasch et al., New J. Phys. 12, 065024 (2010).

[35] S. M. Barnett and P. M. Radmore, Methods in Theoretical Quantum Optics (Oxford University, Oxford, 2002).

[36] G. Vidal and R. F. Werner, Phys. Rev. A 65, 032314 (2002).

[37] M. Hillery and M. S. Zubairy, Phys. Rev. Lett. 96, 050503 (2006); Phys. Rev. A 74, 032333 (2006).

[38] R. Horodecki, P. Horodecki, M. Horodecki, and K. Horodecki, Rev. Mod. Phys. 81, 865 (2009).

[39] G. R. Jin, P. Zhang, Y.-X. Liu, and C. P. Sun, Phys. Rev. B 68, 134301 (2003). 